\title{
Rotating Adjustable Transmission Optical Step Attenuator
}

\author{
Ared Cezairliyan \\ Institute for Materials Research, National Bureau of Standards, Washington, D.C. 20234
}

(March 12, 1971)

\begin{abstract}
A rotating wavelength-independent optical attenuator is described that generates four radiance steps. Radiance ratios between the steps can be continuously varied. Experiments are performed to assess the operational characteristics of the attenuator. Radiance is determined from measurements with a high-speed photoelectric pyrometer. Recording of signals is made with a high-speed digital data acquisition system. The combined measuring and recording systems have a full-scale signal resolution of approximately one part in 8000 and a time resolution of $0.4 \mathrm{~ms}$. The results of several experiments on radiance ratio measurements are found to be in agreement within one part in 1000 .
\end{abstract}

Key words: High-speed measurements; optical attenuator; photometry; radiance.

\section{Introduction}

Rotating step attenuators are used to evaluate the linearity of transmission of optical systems and to calibrate radiation detectors. However, in general they are made of radiation absorbing materials, such as neutral density filters. They have limitations due to the fact that filter transmission is, to some extent, dependent on the wavelength of radiation. In addition, such attenuators are not capable of producing variable radiance ratios between the steps, and they are used for steady-state type calibrations.

In this paper a rotating, wavelength-independent, optical attenuator is described that generates four radiance steps. Radiance ratios between the steps can be continuously varied, and the attenuator can be used for calibrations under dynamic conditions.

\section{Method and Apparatus}

The method of step attenuation is based on varying the aperture stop of an optical system shown in figure 1 . The optical system consists of a radiance source (tungsten filament lamp), a rotating disk, special diaphragms, a high-speed pyrometer, and other pertinent components.

The rotating disk has four circular slits of different widths (fig. 2). Because the simple milling of a slit may not produce an optically smooth edge, a fabrication technique was used in which four grooves of different width and depth were cut on a lathe on one side of a disk. The other side of the disk was then milled to a different depth in each quadrant and to a greater width than the groove. Cross section of the disk at the midplane of each slit is shown in figure 3 . The disk was dynamically balanced.

The diaphragm (D in fig. 1) with a fixed rectangular opening defines the maximum aperture stop of the optical system. There are four individually adjustable diaphragms (fig. 4), which are used to vary the attenuation

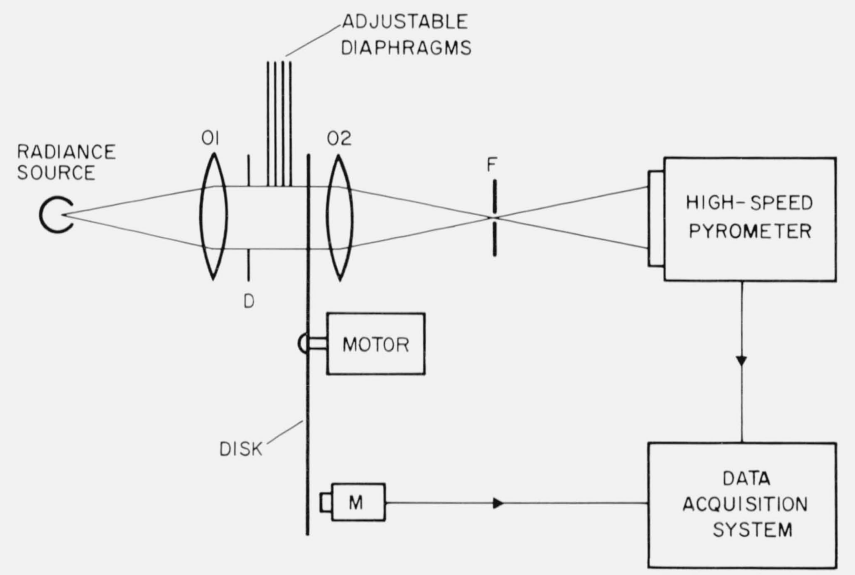

Figure 1. Schematic diagram of optical system for generation of adjustable-amplitude radiance steps.

ratio between the steps. The aperture stop at a given time is defined by the position of the adjustable diaphragms and the particular slit in the optical path. The lenses (01 and 02 in fig. 1) are $25 \mathrm{~mm}$ in diameter with $f / 2.5$.

Rotation of the disk was synchronized with other measuring equipment by a signal obtained from a magnetic pickup. The magnetic pickup M (fig. 1) generated a signal each time a magnetic material embedded in the disk was in line with the pickup.

Relative radiance of the source after attenuation was measured with a high-speed photoelectric pyrometer. ${ }^{1}$ The electrical signals from the pyrometer were recorded with a high-speed digital data acquisition system. ${ }^{2}$ The combined measuring and recording system has a full-scale signal resolution of approximately one part in 8000 and a

\footnotetext{
1 Foley, G. M., Rev. Sci. Instr. 41, 827 (1970).

2 Cezairliyan, A., M. S. Morse, H. A. Berman, and C. W. Beckett, J. Res. Nat. Bur. Stand. (U.S.) 74A (Phys. and Chem.), 65 (1970).
} 
time resolution of $0.4 \mathrm{~ms}$. The photomultiplier in the pyrometer was alternately exposed to the radiance from the tungsten filament lamp and to the radiance from a reference lamp. This scheme eliminated errors that result from photomultiplier fatigue, etc.

A typical oscilloscope trace photograph of the pyrometer output for the case where the adjustable diaphragms are not in the optical path is shown in figure 5. Dots forming the short horizontal lines correspond to radiances from different slits. Dots forming the long horizontal lines correspond to radiances from the reference source.

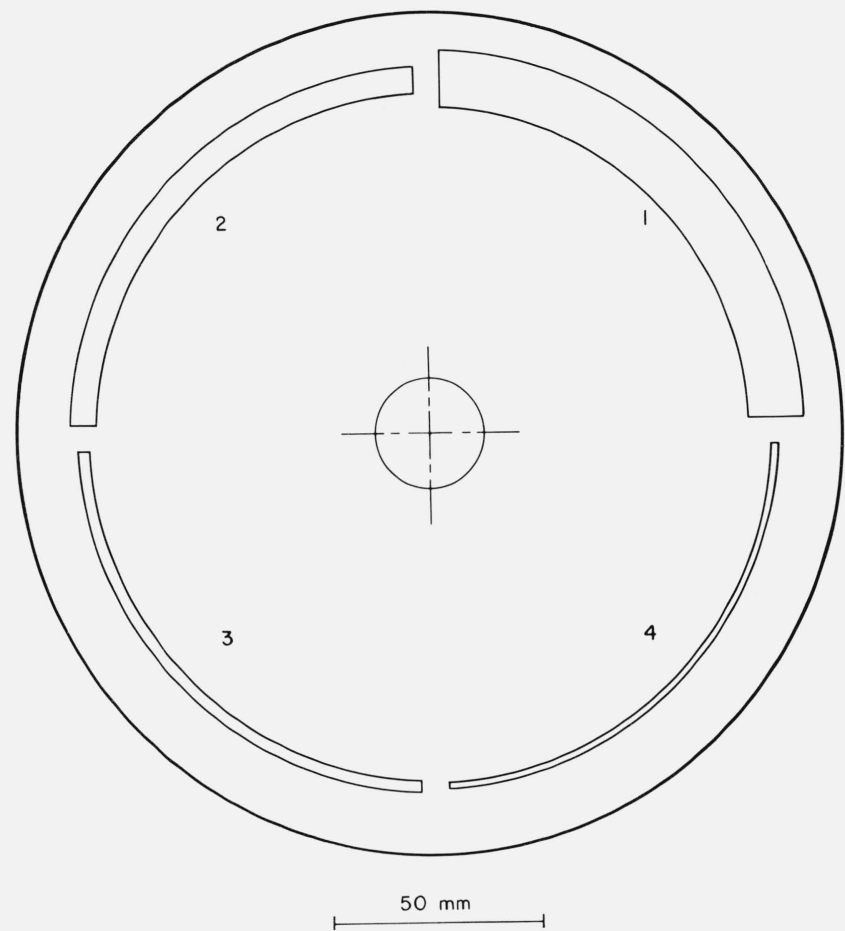

Figure 2. Attenuator disk, showing effective opening of each slit.
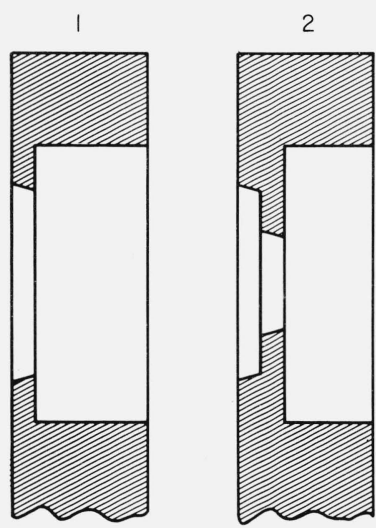

3
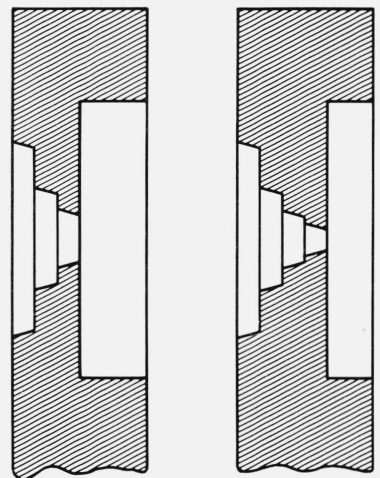

$10 \mathrm{~mm}$

FIGURE 3. Cross section of attenuator disk at midplane of each slit.

Amplitude of the radiance pulses is proportional to the effective aperture stop. For the case in which the adjusta- ble diaphragms are not in the optical path, the ratio of radiances corresponding to different slits is almost equal to the ratio of the slit widths. By inserting different diaphragms in the optical path by different amounts, the radiance ratios can be varied continuously.
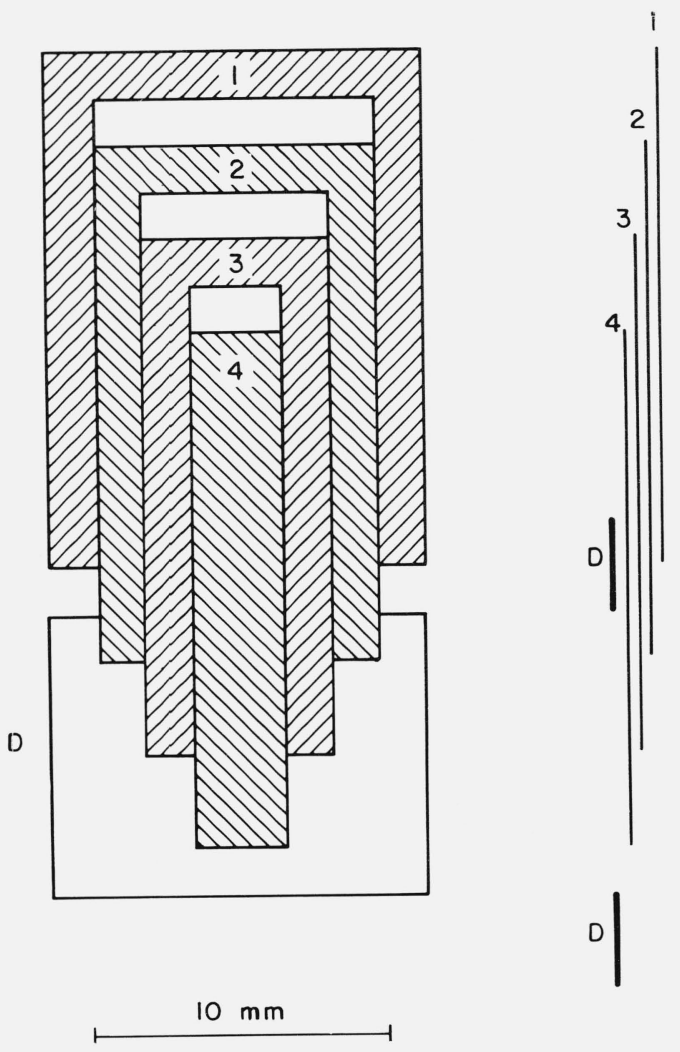

Figure 4. The adjustable diaphragms.

\section{Experimental Results}

In order to assess the operational characteristics of the optical attenuator system several experiments were conducted which are summarized in the following paragraphs.

Four experiments were performed at different disk speeds ranging from 500 to $1800 \mathrm{rpm}$. In all the experiments, the adjustable diaphragms were removed from the optical path. The experiments were similar to the one shown in figure 5 . The experimental quantities obtained using the high-speed digital data acquisition system were processed with a computer.

Radiances obtained from four different slits enable the computation of six radiance ratios, $\tau_{i j}$, which are defined by

$$
\tau_{i j}=\frac{L_{j}}{L_{i}}
$$

where $L_{i}$ and $L_{j}$ are radiances corresponding to slits i and $\mathrm{j}$ respectively.

The ratio of radiances corresponding to various slits are given in table 1. It may be seen that, on the average, the results of four experiments are in agreement with each other within one part in 1000. 


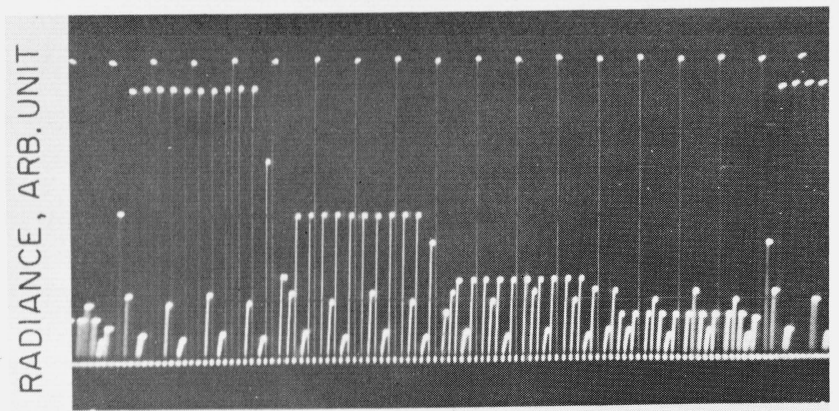

$$
\text { TIME, }
$$

Figure 5. Typical oscilloscope trace photograph of pyrometer output showing the four radiance steps.

TABLE 1. Radiance ratios corresponding to various slit combinations

\begin{tabular}{|c|c|c|c|c|c|c|}
\hline$\tau_{\mathrm{ij}}{ }^{*}$ & $\begin{array}{l}\text { Exp. 1 } \\
\text { (1800 } \\
\text { rpm) }\end{array}$ & $\begin{array}{l}\text { Exp. 2 } \\
\text { (1100 } \\
\text { rpm) }\end{array}$ & $\begin{array}{c}\text { Exp. } 3 \\
(750 \\
\text { rpm) }\end{array}$ & $\begin{array}{l}\text { Exp. } 4 \\
(500 \\
\text { rpm) }\end{array}$ & $\begin{array}{l}\text { Avg. of } \\
\text { Four } \\
\text { Expts. }\end{array}$ & $\begin{array}{c}\text { Avg. } \\
\text { Absolute } \\
\text { Diff. (\%) }\end{array}$ \\
\hline$\tau_{12}$ & 0.5118 & 0.5118 & 0.5114 & 0.5108 & 0.5115 & 0.07 \\
\hline$\tau_{13}$ & .2638 & .2634 & .2637 & .2633 & .2636 & .08 \\
\hline$\tau_{14}$ & .1281 & .1279 & .1276 & .1278 & .1279 & .08 \\
\hline$\tau_{23}$ & .5154 & .5147 & .5156 & .5154 & .5153 & .05 \\
\hline$\tau_{24}$ & .2503 & .2498 & .2497 & .2503 & .2500 & .11 \\
\hline$\tau_{34}$ & .4855 & .4854 & .4840 & .4856 & .4851 & .12 \\
\hline
\end{tabular}

${ }^{*} \tau_{\mathrm{ij}}$ is defined by eq. (1); subscripts refer to slits as marked on figure 2 .

Internal consistency of the results was checked by computing new ratios from a group of directly measured ratios according to the following relations:

$$
\begin{aligned}
\tau_{i j} & =\frac{\tau_{i k}}{\tau_{j k}} \\
\tau_{i j} & =\left(\tau_{i k}\right)\left(\tau_{k j}\right) \\
\tau_{i j} & =\left(\tau_{i k}\right)\left(\tau_{k l}\right) \quad\left(\tau_{l j}\right)
\end{aligned}
$$

For numerical calculations, ratios corresponding to the average of four experiments were used. A comparison of directly measured and computed radiance ratios is given in table 2. It may be seen that the agreement is better than 5 parts in 10,000 .

The uniformity of the slits in the disk was checked under steady-state conditions using the same optical arrangement with the exception that an automatic photoelectric pyrometer was used. A total of 40 measurements were made at different slit positions. The results indicate that average radiance nonuniformity created by nonuniformities of the slits is less than 0.1 percent. The contribution of random noise of the high-speed pyrometer to an individual radiance measurement is approximately 0.2 percent. However, when a large number of points is averaged the reliability of the final result is better than 0.2 percent.

Several experiments were conducted in which the adjustable diaphragms were used to obtain different radiance ratios. Oscilloscope trace photographs of the high-speed pyrometer output are shown in figures 6,7 , and 8 for various diaphragm positions and combinations. In all cases, the individual diaphragms were either completely in or completely out of the optical path. By placing the diaphragms in intermediate positions continuous variation in the radiance ratios can be obtained.

\section{Conclusions}

The results of the experiments described show the feasibility of generating adjustable-amplitude radiance steps. Attenuation of the disk and adjustable diaphragms is independent of wavelength. The radiance ratio between steps can be adjusted continuously. Results of radiance ratio measurements at four disk speeds agree to 1 part in 1000 .

The optical step attcnuating system described in this paper allows determination of the linearity of a radiation detector under dynamic conditions. Because of its versatility in generating radiance pulses, this system may find application in experiments involving high-speed heating or cooling of specimens.

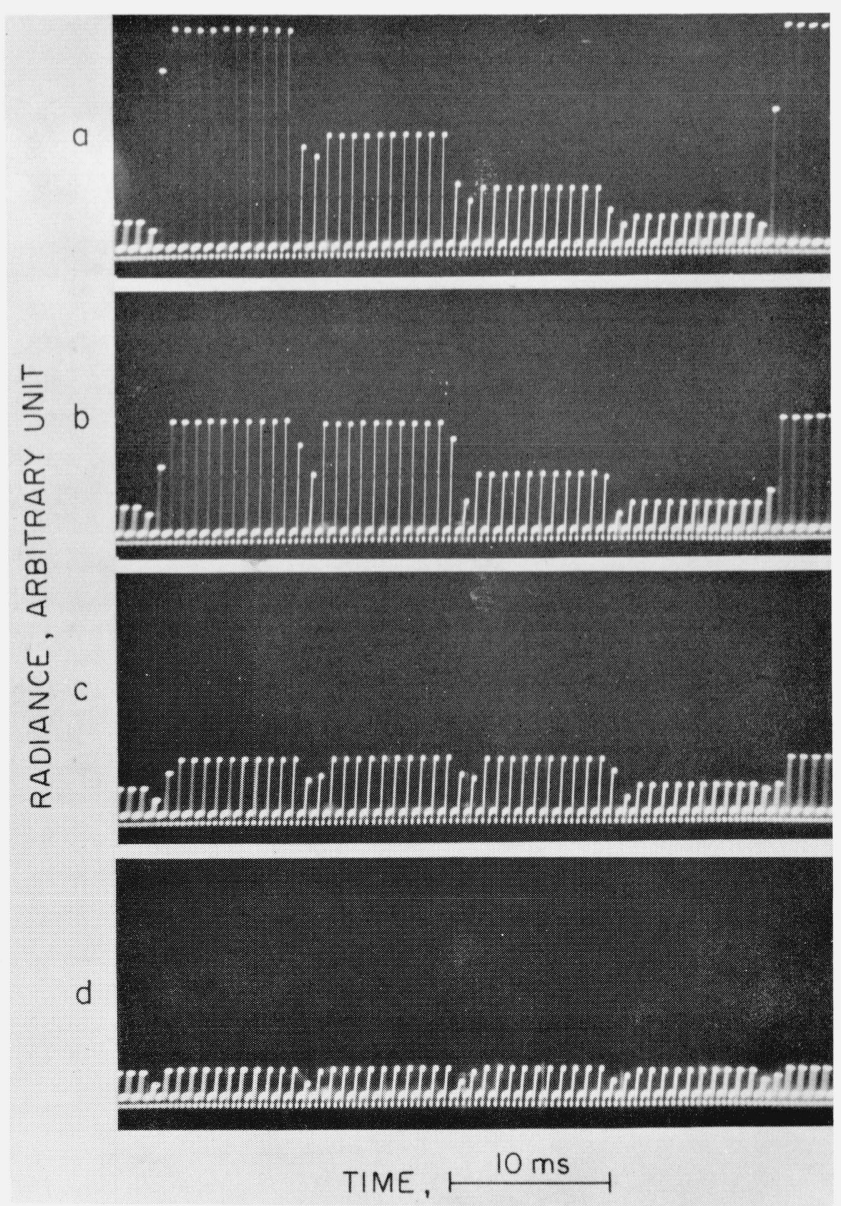

Figure 6. Oscilloscope trace photographs of pyrometer output showing radiance steps corresponding to various adjustable diaphragm settings: (a) no diaphragm in optical path; (b) only diaphragm 1 in; (c) diaphragms 1 and 2 in; (d) diaphragms 1 , 2 , and 3 in. 
TABLE 2. Comparison of radiance ratios determined from direct measurements and from combination of related ratios

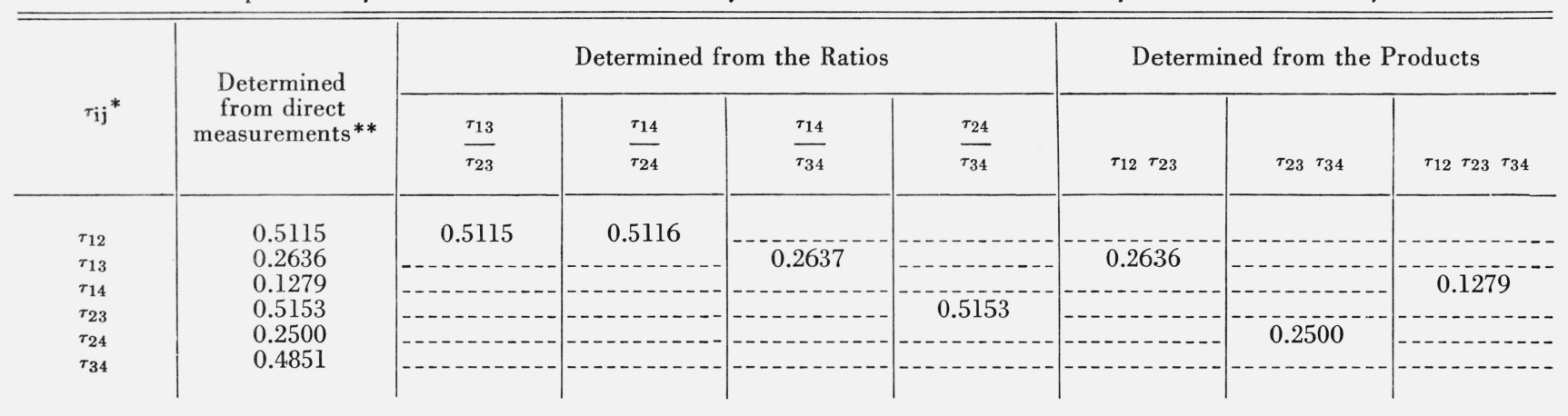

${ }^{*} \tau_{\mathrm{ij}}$ is defined by eq. (1); subscripts refer to slits as marked on figure 2 .

**Averages of four experiments are used.

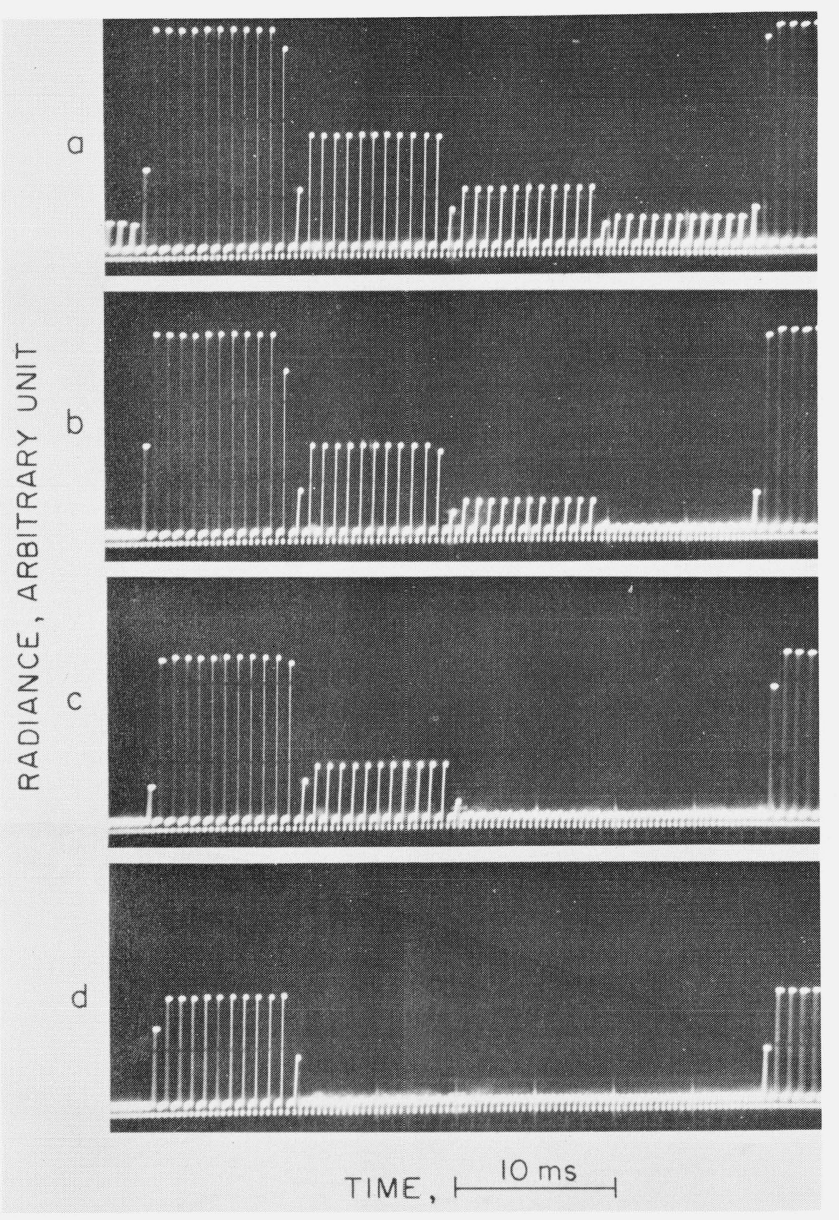

Figure 7. Oscilloscope trace photographs of pyrometer output showing radiance steps corresponding to various adjustable diaphragm settings: (a) no diaphragm in optical path; (b) only diaphragm 4 in; (c) diaphragms 3 and 4 in; (d) diaphragms 2, 3and 4 in. a

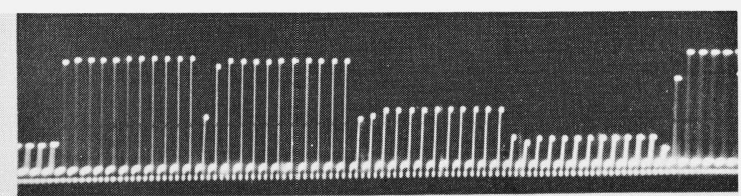

b

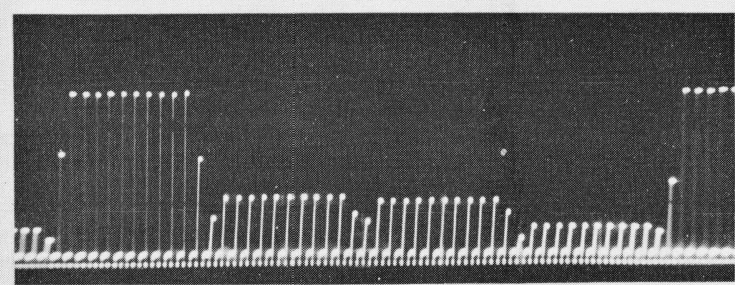

d
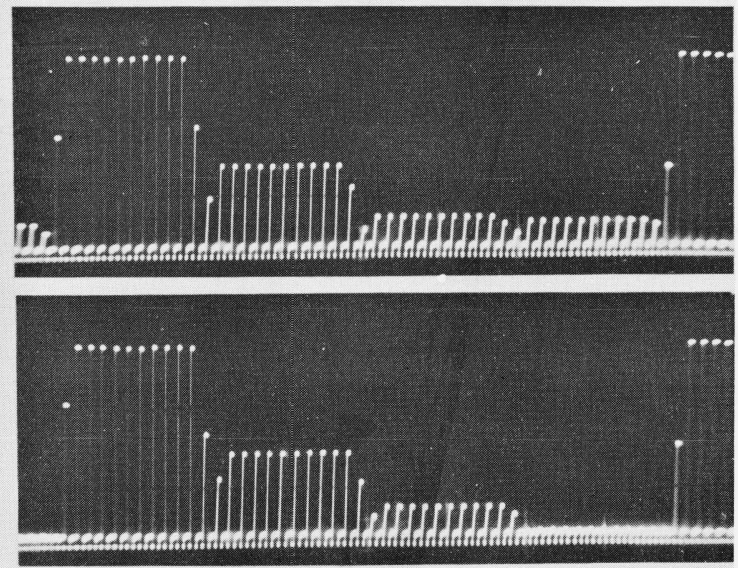

$$
\text { TIME, }
$$

Figure 8. Oscilloscope trace photographs of pyrometer output showing radiance steps corresponding to various adjustable diaphragm settings: (a) only diaphragm 1 in optical path; (b) only diaphragm 2 in; (c) only diaphragm 3 in; (d) only diaphragm 4 in. 